\title{
Editorial \\ Sprachliche Bildung von \\ 3-bis 8-jährigen Kindern
}

\section{Dieter Isler, Sandrine Aeby Daghé und Edina Krompàk}

In der Schweiz besteht bereits beim Schuleintritt ein vergleichsweise starker Zusammenhang zwischen der familiären Herkunft und den sprachlichen Fähigkeiten der Kinder (Stamm, Brandenberg, Knoll, Negrini \& Sabini, 2012; Moser, 2005). Verschiedene Studien bestätigen diesen Befund auch für Deutschland (Niklas, Schmiedeler \& Schneider, 2010; Weinert, Ebert \& Dubowy, 2010) und Frankreich (Bautier, 2001). In den aktuellen bildungspolitischen Diskussionen wird deshalb die Bedeutung der frühen sprachlichen Bildung betont (Grossenbacher, 2010). Der Besuch einer qualitativ guten frühpädagogischen Einrichtung kann den Erwerb sprachlicher Fähigkeiten langfristig unterstützen (Bos, Lankes, Schwippert, Valtin, Voss, Badel \& Plassmeier, 2003; Sammons, Anders, Sylva, Melhuish, Siraj-Blatchford, Taggart \& Barreau, 2008). Dabei sind Bildungseinrichtungen wie Spielgruppen, Kindertagesstätten, Kindergärten oder Grundschulen nicht nur isoliert, sondern auch im Hinblick auf ihre Anschlussfähigkeit an heterogene familiäre Bildungserfahrungen (Lahire, 1993; Künzli, Isler \& Leeman, 2010) sowie an vorausgehende und nachfolgende Einrichtungen (Panagiotopoulou \& Graf, 2008; Seifert 2013) zu betrachten. Eine gemeinsame Aufgabe der vorschulischen und schulischen Bildungsorte besteht darin, möglichst allen Kindern einen altersgerechten Zugang zu sprachlicher Bildung zu verschaffen. Spielgruppen und Kindertagesstätten sind aber ganz anders organisiert als die Schule, und für den Frühbereich, den Kindergarten und die Schule gelten unterschiedliche Bildungsverständnisse und Regelstrukturen (SKBF, 2010; Sörensen \& Thévenaz-Christen, 2003). Um das Ziel einer altersgerechten und anschlussfähigen sprachlichen Bildung zu realisieren, ist es deshalb notwendig, zunächst die bestehenden sprachlichen Bildungsangebote zu verstehen.

Die Beiträge dieser Nummer der Schweizerischen Zeitschrift für Bildungswissenschaften beziehen sich auf die sprachliche Bildung von Kindern im Alter von 3-8 Jahren. Sie befassen sich auf der Grundlage empirischer Untersuchungen mit sprachlichen Fähigkeiten von Kindern in mehrsprachigen Kontexten sowie mit Praktiken der Sprachförderung in verschiedenen Bildungseinrichtungen. Im 
Folgenden werden zunächst einige übergreifende Aspekte beleuchtet, die sich in mehreren Studien als relevant erwiesen haben: die Bedeutung von Genres für die Sprachförderung, unterschiedliche Konstellationen von Mehrsprachigkeit, Prozesse der Reproduktion bzw. des Abbaus von Bildungsungleichheit und das Handeln der pädagogischen Fachpersonen in Interaktionen. Danach geht es um die gewählten methodischen Zugänge. Abschliessend werden die einzelnen Beiträge kurz vorgestellt und kommentiert.

Verschiedene Studien haben die Frage untersucht, wie sprachliche Interaktionen situativ auszugestalten sind, damit sie den Spracherwerb unterstützen (Bautier, 2001; Canut \& Vertalier, 2009). Sie fokussieren darauf, dass Kinder Sprache nicht durch blossen Kontakt erwerben, sondern dass sie sprachliche Mittel in konkreten Anwendungssituationen produktiv anwenden und dabei ihre Funktionen erproben müssen. Für die schulische Sprachförderung stellt das Konzept der "Genres der formellen Mündlichkeit» ("genres oraux formels»; Dolz \& Schneuwly, 1998; Thévenaz-Christen, 2008) einen konzeptionellen Rahmen bereit, der nicht nur der Analyse sprachlicher Praktiken, sondern auch der Förderung textuell-pragmatischer Sprachfähigkeiten dient. Dieser theoretisch-methodologische Zugang berücksichtigt sowohl die durch Kommunikationssituationen und ihre institutionelle Kontexte bedingte Vielfalt von Textproduktionen als auch deren Musterhaftigkeit. Aus dieser Perspektive können Genres wie Phantasieerzählungen, Erlebnisberichte, Erörterungen oder Wegbeschreibungen identifiziert werden. Erst die Kenntnis solcher Muster ermöglicht es, entsprechende Texte funktionsangemessen zu verstehen und zu produzieren. Dieses Konzept ist auch für den frühen Erwerb zweisprachiger Praktiken fruchtbar (s. Sánchez Abchi in diesem Band). Zudem bietet es interessante Ansätze für den immersiven Zweitsprachunterricht (s. Manterola und Almgren in diesem Band).

Während die gesellschaftliche und individuelle Mehrsprachigkeit in den bildungspolitischen Diskussionen überwiegend Wertschätzung und Bedeutung erhält (Allemann-Ghionda, 2013; Gogolin, 2010), gibt es immer noch wenig Anhaltspunkte, wie die individuelle Mehrsprachigkeit im pädagogischen Alltag gefördert werden soll. Darüber hinaus besteht weiterhin eine Forschungslücke bezüglich der sprachlichen Praktiken mehrsprachiger Kinder in vorschulischen und schulischen Bildungsinstitutionen sowie des Umgangs dieser Institutionen mit sprachlicher Diversität. Das Forschungsprojekt HeLiE der Universität zu Köln und dessen Anschlussprojekt MEMOS gehen der Frage über die Qualität der praktizierten Sprachförderung im Kindergarten und im Übergang in die Primarschule nach. Kassis-Filippakou und Panagiotopoulou (in diesem Band) zeigen anhand des Gebrauchs der Varietäten Dialekt und Hochdeutsch auf, dass flexible Mehrsprachigkeit im Kindergarten und in der Unterstufe sowohl vorausgesetzt als auch hervorgebracht wird, und zwar von Lehrpersonen und Kindern. Entsprechend plädieren sie für eine Anerkennung gelebter Mehrsprachigkeit als Realität und Chance. 
Für die Bildungsforschung stellt die institutionelle Selektion insbesondere im Übergang von der vorschulischen in die schulische Institution ein zentrales Thema dar. Die vorliegenden empirischen Studien unterstreichen die Bedeutung dieser Schnittstelle in der Reproduktion von Ungleichheit (Gomolla \& Radke, 2002; Kronig, 2007). Bislang lagen wenige empirische Studien vor, welche die Ungleichheitsgenese auf der Mikroebene untersuchten (vgl. Diehm, Kuhn, Machold \& Mai, 2013). Der Beitrag von Edina Krompàk (in diesem Band) widmet sich der Rolle des Kindergartens bei der Reproduktion von Bildungsungleichheit und untersucht längsschnittlich, mit der ethnographischen Forschungsstrategie den Bildungsweg eines Kindes und dadurch die Selektivität des Bildungssystems in der alltäglichen institutionellen Praxis.

Die konkrete Ausgestaltung von Interaktionen zwischen Fachpersonen und Kindern ist als Prozessqualität von zentraler Bedeutung für eine wirksame Sprachförderung in pädagogischen Einrichtungen (Henry \& Pianta, 2011; Kuger \& Kluczniok, 2008). Bisher lagen zum interaktiven Handeln von pädagogischen Fachpersonen in Deutschschweizer Spielgruppen, Kitas und Kindergärten im Hinblick auf Sprachförderung keine Untersuchungen vor. In dieser Nummer werden drei aktuelle Forschungsarbeiten vorgestellt, die auf dieses dringliche Desiderat reagieren und - mit unterschiedlichen Fokussierungen und methodischen Zugängen - spracherwerbsunterstützende Potenziale von Alltagsgesprächen untersuchen. Die Ergebnisse dieser drei Studien tragen gemeinsam zu einer substanziellen Ausdifferenzierung des Untersuchungsgegenstands (des Handelns pädagogischer Fachpersonen bei der Sprachförderung) bei: Wiesner und Isler beleuchten den Zusammenhang zwischen Konzeption und Realisierung von Bildungsprozessen und zeigen, wie Lehrpersonen durch multimodale Unterstützungssysteme, unterschiedliche Beteiligungsrollen und zugeschnittene Aufgabenstellungen die Teilhabe aller Kinder erleichtern. Kannengieser und Tovote entwickeln auf der Basis ihrer Beobachtungen eine Typologie von zwölf Interaktionsarten und identifizieren drei Grunddimensionen zur Reflexion und Planung sprachförderlicher Interaktionen. Vogt, Löffler, Haid, Itel, Schönfelder, Zumwald und Reichmann belegen die Relevanz verschiedener Sprachförderstrategien im pädagogischen Alltag und untermauern die Forderung nach vermehrter Realisierung längerer, dialogisch-entwickelnder Gespräche. Zudem verweisen sie auf die institutions- aber auch personenspezifische Prägung von Interaktionsstilen.

Den unterschiedlichen Gegenständen und Fragestellungen entsprechend kamen in den berichteten Studien verschiedene Designs und Methoden zum Einsatz. Die Untersuchungen basieren auf längsschnittlichen, pseudo-längsschnittlichen und einmaligen (teils zeitintensive) Erhebungen. Die Beobachtungen sind als Feldnotizen, Textdokumente, Audio- und Videoaufnahmen dokumentiert. Das Spektrum der Auswertungsverfahren reicht von linguistische Korpusanalysen über deduktive und induktive Kodierverfahren - verbunden mit statistischen oder konzeptentwickelnden Auswertungen - bis zu sequenzanaly- 
tischen Rekonstruktionen. Diese unterschiedlichen Zugänge zum Gegenstand der frühen Sprachförderung ergeben ein facettenreiches Bild und interessante Möglichkeiten für methodologische Vergleiche (die an dieser Stelle allerdings nicht geführt werden können).

Nun zu den einzelnen Beiträgen dieser Nummer. Sie sind ihren Gegenständen und Zugangsweisen entsprechend wie folgt gruppiert: Zunächst werden zwei Untersuchungen vorgestellt, die sich auf der Grundlage von Sprachproduktionen mit Genrekompetenzen von Kindern in mehrsprachigen Kontexten befassen (Sanchez Abchi und Manterola \& Almgren). Danach geht es um Studien, die das Handeln von pädagogischen Fachpersonen in verschiedenen Bildungseinrichtungen mittels Interaktionsanalysen untersuchen (Kannengieser \& Tovote, Wiesner \& Isler und Vogt et al.). Abschliessend werden zwei ethnografische Arbeiten zu Praktiken der Sprachverwendung und Selektion präsentiert (Kassis \& Panagiotopoulou, Krompák).

Verónica Sánchez Abchi befasst sich mit den sprachlichen Fähigkeiten von zweisprachig (spanisch und schweizerdeutsch) aufwachsenden Kindern beim produzieren von mündlichen Erlebnisberichten. Die beobachteten Unterschiede der zweisprachigen Kinder im dritten und fünften Lebensjahr gleichen jenen der einsprachig (schweizerdeutsch) aufwachsenden Kinder aus der Kontrollgruppe. Die älteren Kinder sind beim Berichten ihrer Erlebnisse selbständiger als die jüngeren, sie verwenden aber auch mehr schweizerdeutsche Wörter, um ihre Texte weiter zu entwickeln. Die Autorin interpretiert die zunehmende Verwendung schweizerdeutscher Wörter nicht nur als Problem des fehlenden spanischen Wortschatzes, sondern auch als Strategie zur Anreicherung ihrer Berichte und zur Aufmerksamkeitslenkung der Zuhörenden und damit als Potenzial der Mehrsprachigkeit dieser Kinder.

Ibon Manterola und Margareta Almgren untersuchen den Erwerb des Baskischen als Zweitsprache in Kontext eines Förderprogramms zur Wiederbelebung dieser Sprache durch immersiven Unterricht. Sie zeigen, dass lexikalische Schwierigkeiten im Baskischen, wie sie bei den fünfjährigen Kindern beobachtet wurden, auch im Alter von acht Jahren noch bestehen, wobei die Grenzen zwischen den beiden Sprachen durchlässiger und die Bezüge flexibler werden. Die Kinder, die Baskisch als familiäre Erstsprache benutzen, unterscheiden sich von der Gruppe, die Baskisch als Zweitsprache lernt, durch Abweichungen von der Standardform des Baskischen sowie durch die bessere Beherrschung der indirekten Rede und des Ergativs (eines besonderen Falls des Baskischen).

Kannengieser und Tovote berichten über die Teilergebnisse der Studie «MeKi - Die frühe Förderung mehrsprachiger Kinder ab 3 Jahren» zum Thema alltagsintegrierte Sprachförderung in der Spielgruppe. Im Fokus der Teilstudie standen die Interaktionen zwischen den Fachpersonen und den einzelnen Zielkindern und insbesondere die Spachlerngelegenheiten im untersuchten Setting. Die Analyse der Interaktionssequenzen zeigte zwölf verschiedene Interaktionstypen, die entlang der Dimensionen «Kommunikationszweckorientierung vs. Bezie- 
hungsorientierung», «Unidirektionalität vs. Bi-bzw. Multidirektionalität» sowie «Gegenwartsbezogenheit vs. Vergegenwärtigung» eingeordnet werden können. Die vorgestellte Studie liefert qualitative Informationen über die Grundvarianten der Interaktionen, mit deren Hilfe die situative Fördergelegenheiten in der pädagogischen Praxis identifiziert und reflektiert werden können.

Esther Wiesner und Dieter Isler stellen Ergebnisse des Forschungsprojekts «Prozesse der Sprachförderung im Kindergarten -ProSpiK» in Bezug auf die multimodale Unterstützung des Erwerbs sprachlich-kognitiver Fähigkeiten dar. Sie untersuchen die kommunikativen Formen des bildungssprachlichen Handelns und deren interaktive Herstellung durch Lehrpersonen und Kinder. Aufgrund der Sequenzanalyse von Videodaten rekonstruieren sie ein didaktisches Setting, in dem die Kinder lernen, den eigenen Standpunkt kognitiv, räumlich-körperlich und verbal einzunehmen. Dieses Setting und seine multimodale Ausgestaltung eröffnen den Kindern unterschiedliche Beteiligungsmöglichkeiten im Unterricht. Aufgrund der Ergebnisse der Studie wird die Bedeutung der gezielten Förderung komplexer Sprachhandlungen wie z.B. Argumentieren betont. Darüber hinaus weisen Wiesner und Isler auf die Notwendigkeit hin, dass sich Lehrpersonen in der Aus- und Weiterbildung sowohl mit der Planung als auch mit der interaktiven Realisierung didaktischern Settings auseinandersetzen sollten.

Franziska Vogt, Cordula Löfler, Andrea Haid, Nadine Itel, Mandy Schönfelder, Bea Zumwald und Elke Reichmann untersuchen das Handeln pädagogischer Fachpersonen in drei unterschiedlichen Einrichtungen - Spielgruppen, Kindertagesstätten und Kindergärten - im Hinblick auf die sprachfördernde Ausgestaltung von Alltagsgesprächen. Der Fokus ihrer Studie liegt auf der Anwendung von anerkannten Sprachförderstrategien und auf der Wirksamkeit einer darauf ausgerichteten Weiterbildungsmassnahme. Zu diesem Zweck haben sie Alltagsgespräche gefilmt und anhand eines deduktiv entwickelten Kategoriensystems untersucht. In diesem Beitrag vergleichen sie drei Fälle aus den drei unterschiedlichen institutionellen Kontexten. Dabei bestätigt sich u.a., dass dialogisch-entwickelnde Gespräche nur selten vorkommen. Zudem zeigen sich sowohl institutionell als auch individuell geprägte Gesprächsstile der pädagogischen Fachpersonen.

Maria Kassis-Filippakou und Argyro Panagiotopoulou befassen sich in ihrer ethnografischen Studie mit Mehrsprachigkeit im Unterricht am Übergang vom Kindergarten in die erste Primarklasse. Im vorliegenden Beitrag fokussieren sie den Gebrauch der Varietäten Dialekt und Standard. Anhand von audiografisch dokumentierten Unterrichtsgesprächen zeigen sie auf, dass diese Varietäten in der alltäglichen Unterrichtskommunikation nicht nur von den Kindern, sondern auch von Lehrpersonen flexibel eingesetzt werden. Sie plädieren dafür, Mehrsprachigkeit als Normalität anzuerkennen und zu fördern und damit der Problematisierung des Sprachenmischens sowie der Exotisierung weiterer Familiensprachen entgegenzuwirken. 
Auch Edina Krompák befasst sich aus einer ethnografischen Perspektive mit dem Übergang vom Kindergarten in die erste Primarklasse. In ihrem Beitrag nimmt sie das Handeln pädagogischer Fachpersonen im Zusammenhang mit Übertrittsentscheidungen in den Blick. Gestützt auf Unterrichtsbeobachtungen, Dokumentanalysen und Gespräche mit Lehrpersonen, Kindern und Eltern zeigt sie, dass nicht so sehr die sprachlich-kognitiven Fähigkeiten, sondern die Konformität mit der Rolle des Schülerin bzw. des Schülers über den Eintritt in die erste Klasse entscheiden. Zudem wird in beklemmender Weise deutlich, wie das Mitspracherecht von Eltern zwar scheinbar respektiert, letztlich aber unterlaufen wird.

Die Beiträge dieser Nummer zeigen, dass der Erwerb sprachlicher Fähigkeiten nur unter Einbezug der situativen, institutionellen und (sprach-)kulturellen Kontexte angemessen verstanden werden kann. Die vorgestellten Arbeiten skizzieren unterschiedliche Perspektiven, die aber darin übereinstimmen, dass Rollen (von Lehrenden und Lernenden), Sprachen (Erst-, Zweit- und Fremdsprachen, Varietäten und Modalitäten) und Institutionen (wie Familien, Spielgruppen, Kitas und Schulen) nicht isoliert, sondern in ihrem komplexen Zusammenspiel untersucht werden müssen. Das Koordinationsteam wünscht Ihnen eine interessante Lektüre.

\section{Literatur}

Allemann-Ghionda, C. (2013). Bildung für alle, Diversität und Inklusion: Internationale Perspektiven. Padernborn: Schöningh.

Bautier, E. (2001). Pratiques langagières et scolarisation. Note de synthèse. Revue française de pédagogie, 137, 117-141.

Bos, W., Lankes, E., Schwippert, K., Valtin, R., Voss, A., Badel, I. \& Plassmeier, N. (2003). Lesekompetenzen deutscher Grundschülerinnen und Grundschüler am Ende der vierten Jahrgangsstufe im internationalen Vergleich. In W. Bos, E. Lankes \& K. Schwippert (Hrsg.), Erste Ergebnisse aus IGLU (S. 69-142). Münster: Waxmann.

Canut, E., \& Vertalier, M. (2009). L'apprentissage du langage, une approche interactionnelle: réflexions théoriques et pratiques de terrain: mélanges offerts par ses collègues, ses élèves et ses amis en hommage à Laurence Lentin. Paris: L'Harmattan.

Diehm, I., Kuhn, M., Machold C., \& Mai, M. (2013). Ethnische Differenz und Ungleichheit. Zeitschrift für Pädagogik, 5, 644-656.

Dolz, J., \& Schneuwly, B. (1998). Pour un enseignement de l'oral. Initiation aux genres formels à l'école. Paris: ESF.

Gogolin, I. (2010). Mehrsprachigkeit. Zeitschrift für Erziehungswissenschaft, 13(4), 529-547.

Gomolla, M., \& Radtke, F.-O. (2002). Institutionelle Diskriminierung. Die Herstellung ethnischer Differenz in der Schule. Opladen: Leske \& Budrich.

Grossenbacher, S. (2010). Das Lebensspannenkonzept zur Prävention und Bekämpfung von Illettrismus. In A. Sturm (Hrsg.), Literales Lernen von Erwachsenen im Kontext neuer Technologien. Münster: Waxmann.

Henry, A., \& Pianta, R. (2011). Effective Teacher-Child Interactions and Childrens Literacy: Evidence for Scalable, Aligned Approaches to Professional Development. In S. Neuman \& D. Dickinson (Eds.), Handbook of Early Literacy Research, Volume 3 (pp. 308-321). New York: Guilford Press. 
Kronig, W. (2007). Die systematische Zufälligkeit des Bildungserfolgs. Theoretische Erklärungen und empirische Untersuchungen zur Lernentwicklung und zur Leistungsbewertung in unterschiedlichen Schulklassen. Bern: Haupt.

Kuger, S., \& Kluczniok, K. (2008). Prozessqualität im Kindergarten - Konzept, Umsetzung und Befunde. In H. Roßbach \& H. Rossfeld (Hrsg.), Frühpädagogische Förderung in Institutionen (S. 159-177). Wiesbaden: VS Verlag für Sozialwissenschaften (Zeitschrift für Erziehungswissenschaft, Sonderheft 22 / 2008).

Künzli, S., Isler, D., \& Leemann, R. (2010). Frühe Literalität als soziale Praxis - Analyse von Mikroprozessen der Reproduktion von Bildungsungleichheit. Zeitschrift für Soziologie der Erziehung und Sozialisation, 30(1), 60-73.

Lahire, B. (1993). Culture écrite et inégalités scolaires. Lyon: Presses universitaires de Lyon.

Moser, U. (2005). Lernvoraussetzungen in Schulklassen zu Beginn der 1. Klasse. In U. Moser, M. Stamm \& J. Hollenweger (Hrsg.), Für die Schule bereit? Lesen, Wortschatz und Mathematik und soziale Kompetenzen beim Schuleintritt (S. 167-185). Oberentfelden: Sauerländer.

Niklas, F., Schmiedeler, S., \& Schneider, W. (2010). Heterogenität in den Lernvoraussetzungen von Vorschulkindern. Zeitschrift für Grundschulforschung. Bildung im Elementarund Primarbereich, 3(1), 18-31.

Panagiotopoulou, A., \& Graf, K. (2008). Umgang mit Heterogenität und Förderung von Literalität im Elementar- und Primarbereich im europäischen Vergleich. In B. Hofmann \& R. Valtin (Hrsg.), Checkpoint Literacy. Tagungsband zum 15. Europäischen Lesekongress 2007 in Berlin (S. 110-122). Berlin: Deutsche Gesellschaft für Lesen und Schreiben.

Sammons, P., Anders, Y., Sylva, K., Melhuish, E., Siraj-Blatchford, I., Taggart, B., \& Barreau, S. (2008). Children's Cognitive Attainment and Progress in English Primary Schools During Key Stage 2: Investigating the potential continuing influences of pre-school education. In H. Roßbach \& H. Blossfeld (Hrsg.), Frühpädagogische Förderung in Institutionen. Zeitschrift für Erziehungswissenschaft, Sonderheft 22, 179-197.

Seifert, A. (2013). Die Bedeutung der sprachlich-kulturellen Differenz im Übergang vom Kindergarten in die Grundschule. In E. Wannack, S. Bosshart, A. Eichenberger, M. Fuchs, E. Hardegger \& S. Marti (Hrsg.), 4- bis 12-Jährige. Ihre schulischen und ausserschulischen Lern- und Lebenswelten (S. 63-69). Münster: Waxmann.

SKBF Schweizerische Koordinationsstelle für Bildungsforschung (2010). Bildungsbericht Schweiz 2010. Aarau: Schweizerische Koordinationsstelle für Bildungsforschung.

Sörensen, B. \& Thévenaz-Christen, T. (2003). Editorial zum Thema «Kleinkinder- und Vorschulbildung: Praktiken und Ziele». Schweizerische Zeitschrift für Bildungswissenschaften, 25(2), 207-210.

Stamm, M., Brandenberg, K., Knoll, A., Negrini, L. \& Sabini, S. (2012). FRANZ. Früher an die Bildung - erfolgreicher in die Zukunft? Familiäre Aufwachsbedingungen, familienergänzende Betreunng und kindliche Entwicklung. Schlussbericht. Freiburg: Universität Freiburg.

Thévenaz-Christen, T. (2008). Au cour de la forme scolaire, la discipline. L'exemple du français parlé. Schweizerische Zeitschrift für Bildungswissenschaften, 30(2), 299-324.

Weinert, S., Ebert, S. \& Dubowy, M. (2010). Kompetenzen und soziale Disparitäten im Vorschulalter. Zeitschrift für Grundschulforschung. Bildung im Elementar- und Primarbereich, 3(1), 32-45. 
